\title{
VARIABLES QUE DEFINEN EL PERFIL DEL INMIGRANTE UNIVERSITARIO
}

\author{
Gloria Pérez Serrano \\ Ma Luisa Sarrate Capdevila \\ UNED
}

RESUMEN: La diversidad cultural constituye una característica que define a la sociedad actual. Las migraciones se han convertido en los últimos años en una constante atemporal que exige la búsqueda de mecanismos adecuados para gestionar esta nueva realidad, sin producir rupturas ni enfrentamientos. Cabe afirmar que el encuentro de distintas culturas en un mismo espacio geográfico es hoy un hecho estructural. Los organismos internacionales apuestan por promover la convivencia en realidades pluriculturales y luchar para el reconocimiento de los derechos de las minorías. La Universidad, como institución de educación superior, tiene como misión formar a profesionales con un alto nivel de cualificación y ésta debe hacerse extensiva a la población foránea.

El objetivo de este trabajo es definir el perfil del inmigrante universitario, a fin de identificar sus rasgos diferenciales. Se ha utilizado una metodología cuantitativa y cualitativa. Se aplicó un cuestionario de escala tipo Licker así como una entrevista semiestructurada. Se realizaron análisis de los datos utilizando distintos paquetes informáticos.

Entre sus principales resultados destaca que dicha población elige la universidad para continuar su formación, mejorar su empleo y conseguir legalizar su título. Prefieren estudios de larga duración para tener más oportunidades en un mercado laboral competitivo. Sus expectativas son elevadas en orden a mejorar la calidad de vida. Concluye el trabajo aportando medidas de discriminación positiva para favorecer la inclusión social de esta población.

ABSTRACT: Cultural diversity is a defining characteristic of current society. Migrations have in recent years become a timeless constant, a constant that requires a search for mechanisms that can cope with this new reality without triggering rifts or confrontations. It may be asserted that the meeting of different cultures in the same geographic space is today a structural fact. International organizations are committed to promoting living together in pluricultural 
realities and fighting for the acknowledgement of minority rights. The university, as an institution of higher education, has as its mission the training of highly qualified professionals, and this mission must be made to extend to the foreign population.

The objective of this paper is to define the profile of the immigrant university student in order to identify his or her differential traits. A quantitative and qualitative methodology has been used. A Likert-scale questionnaire and a semi-structured interview were applied. The data were analyzed using different computer packages.

Foremost among the main results is the fact that the population at issue chooses to attend university in order to continue their education, improve their job situation and legalize their certificates of educational accomplishment. They prefer long-term studies in order to have more opportunities in a competitive job market. Their expectations are high, aimed at improving their quality of life. The paper concludes with the contribution of positive discrimination measures to favour the social inclusion of this population.

PALABRAS CLAVE: Inmigrante, universidad, perfil, inclusión social, medidas de apoyo.

KEYWORDS: Immigrant, university, profile, social inclusion, supportive measures.

La diversidad cultural e inclusión social es una preocupación relevante de la sociedad actual, que está dando pasos en la búsqueda de modelos y nuevas estrategias de acción socioeducativa, al tiempo que intenta proporcionar una respuesta adecuada a los intereses, necesidades y demandas de los inmigrantes.

Trabajar el tema de la inmigración, requiere una mentalidad abierta y respetar las nuevas formas de comportamiento que se presentan. Este nuevo panorama social demanda cada vez con más fuerza inversiones, tanto en formación como en investigación. La sociedad es y seguirá siendo cada vez más multicultural, por lo que se hace imprescindible encontrar los mecanismos más adecuados para gestionar esta nueva realidad, sin producir rupturas ni enfrentamientos.

Tras una primera etapa en que los países de acogida actuaron de forma etnocéntrica, marginando a las minorías, en la actualidad está emergiendo una mayor conciencia social a favor de la necesidad de luchar para que sus derechos sean reconocidos, en beneficio de una convivencia más justa para todos, lo que genera importantes retos a las políticas culturales, sociales y educativas.

El fenómeno de las migraciones no es nuevo, sino más bien una constante atemporal en todas las sociedades desarrolladas. El Informe de las Naciones Unidas sobre el Desarrollo Humano "Superando barreras: Movilidad y desarrollo humanos" (PNUD, 2009) analiza la migración en el contexto de los cambios y las tendencias demográficas, tanto en términos de crecimiento como de desigualdad. En él se indica que el $3 \%$ de la población mundial es migrante, lo que afecta a 188 millones de personas. 
Cabe afirmar que el encuentro de distintas culturas en un mismo espacio geográfico es hoy en día un hecho estructural y no excepcional. En España y en Europa, esta realidad social está tomando una dimensión especialmente relevante, que exige medidas específicas que promuevan la igualdad de oportunidades y la justicia social. Las repercusiones son importantes en todos los órdenes y el ámbito educativo no es ajeno a las mismas. Cada vez resulta más facil comprobar que las instituciones formativas incluidas las de educación superior, van dejando de ser monoculturales para pasar a convertirse en multiculturales, al acoger a estudiantes de diferentes nacionalidades.

El Informe de la UNESCO coordinado por Delors "Aprender para el siglo XXI. La educación encierra un tesoro" (1996), subraya que la clave para el desarrollo de un país es la educación y la formación a lo largo de la vida. Al mismo tiempo señala, como pilar de la educación para este siglo, la necesidad de aprender a vivir juntos, a convivir con los demás, desarrollando la comprensión del otro y la percepción de las diferentes formas de interdependencia, respetando los valores de pluralismo, comprensión mutua y paz.

Estas razones son más que suficientes para justificar el diseño de una educación inclusiva que permita evitar los conflictos o, si ya se han producido, solucionarlos de manera eficaz. Con ella se fomentará el conocimiento de los demás y de sus culturas, ya que la inclusión ayuda a conciliar los principios de igualdad y de diversidad. Una de sus misiones consiste en mostrar de relieve la riqueza de las semejanzas y diferencias de las distintas realidades para integrarlas en el ejercicio de una ciudadanía responsable.

Esta temática presenta connotaciones importantes en el contexto europeo y no menos en nuestro país en el que va adquiriendo una dimensión más acusada, al irse incrementando, poco a poco, el número de alumnos inmigrantes en todos los niveles educativos. La universidad, en tanto institución de educación superior, debe tener prevista esta situación y diseñar metodologías y nuevas herramientas de discriminación positiva que favorezcan la inclusión social de esta población. Ha de tenerse igualmente en cuenta, que nuestro país demanda personal cada vez más cualificado, por lo que dicha institución educativa está llamada a desempeñar un papel relevante en la formación superior de los inmigrantes y a contribuir, de este modo, a su promoción sociolaboral.

La política europea sobre educación e inmigración tiene como objetivo prioritario lograr la igualdad de oportunidades para todos los hombres y las mujeres que viven y trabajan en su territorio. Todos los países han puesto especial empeño en ello, ya que desde los años ochenta la llegada de inmigrantes no ha dejado de crecer. En la Cumbre de Lisboa (2000), se insistió en la necesidad de que los Estados miembros respondieran a los desafíos planteados a sus sistemas educativos, para hacer frente a la creciente cifra de alumnos procedentes de horizontes muy diversos. Todavía queda pendiente mucha labor por desarrollar puesto que, por el momento, los resultados del proceso de integración por medio de la educación y del trabajo no han Ilegado a alcanzar las expectativas deseadas.

El Informe publicado en el 2007 sobre "Previsiones demográficas mundiales" de la División de Población del Departamento de Asuntos Económicos y Sociales de las 
Naciones Unidas, sitúa a España a la cabeza de los países de la cuenca mediterránea que han pasado de ser importantes emisores de población emigrante a configurarse como destino de una parte creciente de la inmigración.

Según el Padrón Municipal de 2007, el total de residentes en España a 1 de enero de dicho año, era de 45,12 millones. De este total, 40,63 millones poseían la nacionalidad española, en tanto que 4,48 millones, eran extranjeros lo que supone el $11,2 \%$. Esta última cifra incluye a 1,7 millones procedentes de países de la Unión Europea. Algo más de la mitad (el 54,1\%) provienen de Marruecos, Ecuador, Rumania y Colombia, aunque se aprecian aumentos significativos en colectivos como el búlgaro y el boliviano, hasta ahora prácticamente desconocidos.

La legislación española, está intentado responder a esta realidad tal y como se refleja en el artículo 13.1 de la Constitución de 1978. Recientemente, ha surgido la necesidad de adaptarnos a los acuerdos tomados en la Unión Europea en esta materia. Este hecho ha influido en la promulgación de la Ley Orgánica 4/2000, de 11 de enero sobre derechos y libertades de los extranjeros en España y su integración social, normativa que reconoce expresamente, en su artículo 9, el derecho a la educación, incluida la universitaria.

En esta misma línea, el Consejo de Ministro de 16 de febrero de 2007 aprobó el "Plan Estratégico de Ciudadanía e Integración, 2007-2010", documento que cuenta con el informe favorable del Foro para la Integración Social de los Inmigrantes y la aprobación de la Comisión Interministerial de Extranjería y del Consejo Superior de Política de Inmigración. Este Plan se dirige al conjunto de la población, tanto autóctona como extranjera. Está orientado a potenciar la cohesión social a través del fomento de políticas públicas, basadas en la igualdad de derechos y deberes, la igualdad de oportunidades, el desarrollo de un sentimiento de pertenencia a la sociedad española y el respeto a la diversidad. Los principios que lo orientan son los de: igualdad, ciudadanía e interculturalidad. Atención especial recibe la educación al subrayar, entre sus objetivos, la exigencia de adecuarla a las nuevas necesidades que origina la presencia de inmigrantes y de garantizar el acceso a la misma.

Según datos del Consejo de Coordinación Universitaria (2007), en dicho año se graduaron en la universidad española un total de 187.531 estudiantes, de los cuáles 2.268 eran extranjeros. De ellos, 649 corresponden a los países de la Unión Europea y 1.619 al resto; es decir, que de este colectivo de inmigrantes que ha alcanzado una titulación universitaria, un 28,6\% proceden de países de la Unión Europea y un $71,4 \%$ de países no comunitarios. Otro dato indica que una gran parte de los graduados proceden de la Universidad Nacional de Educación a Distancia (UNED). La siguiente tabla refleja la distribución de los estudiantes foráneos por Comunidades Autónomas. 
Tabla 1. Inmigrantes universitarios de $1^{\circ}$ y $2^{\circ}$ Ciclo.

\begin{tabular}{|l|c|c|}
\hline & Total & \% \\
\hline Total de alumnos inmigrantes & 29.627 & 2.1 \\
\hline Universidades Presenciales & 26.754 & 2.2 \\
\hline Andalucía & 4.690 & 2.1 \\
\hline Aragón & 362 & 1.1 \\
\hline Asturias (Principado de) & 158 & 0.6 \\
\hline Balears (Illes) & 436 & 3.3 \\
\hline Canarias & 1.257 & 2.8 \\
\hline Cantabria & 100 & 0.9 \\
\hline Castilla y León & 2.080 & 2.5 \\
\hline Castilla La Mancha & 178 & 0.7 \\
\hline Cataluña & 5.192 & 3.0 \\
\hline Extremadura & 197 & 0.8 \\
\hline Galicia & 864 & 1.2 \\
\hline Madrid (Comunidad de) & 6.424 & 2.8 \\
\hline Murcia (Región de) & 464 & 1.2 \\
\hline Navarra (Comunidad Foral de) & 365 & 2.3 \\
\hline País Vasco & 445 & 0.8 \\
\hline Rioja (La) & 5.489 & 0.8 \\
\hline Valenciana (Comunidad) & 2.882 & 2.5 \\
\hline Universidades No Presenciales & 620 & 1.6 \\
\hline U.N.E.D. & & 0.6 \\
\hline Oberta de Catalunya & 353 & \\
\hline
\end{tabular}

Nota. Información extraída de la publicación Datos y Cifras del Sistema Universitarios Español. Curso 2006-07 del Consejo de Coordinación Universitaria (2007).

Se debe resaltar que el número de universitarios extranjeros matriculados está creciendo año tras año en nuestro país. Es de esperar que este aumento sea constante ya que en el curso 1995-96 la población inmigrante universitaria sólo representaba el $0,68 \%$ del total, en tanto que en 2006-07 ya alcanzó la cifra del 1,94\%, esto es 29.627 alumnos extranjeros, sin incluir los estudiantes Erasmus. La misma tendencia se aprecia en los datos provisionales ofrecidos por el Ministerio sobre el sistema universitario español 2008/2009. La cifra de extranjeros alcanza los 32.136, lo que representa el 2,3\% del total de la población universitaria.

En este artículo se reflejan parte de los resultados de una investigación llevada a cabo con el título "Hacia una universidad inclusiva. Medidas de apoyo al alumna- 
do inmigrante" subvencionada por el Ministerio de Ciencia e Innovación 2008 (Programa de Estudios y Análisis. № Ref. EA-2008-0030) y elaborada por un grupo de investigación con amplia trayectoria en este tema. Partiendo de los datos anteriores y tomando como referencia la figura del inmigrante universitario, el estudio realizado ha perseguido una doble finalidad. Por una parte, identificar las variables que inciden en la inclusión de los inmigrantes en la universidad y su incidencia en el éxito/fracaso académico y, por otra, diseñar medidas de apoyo que propicien su integración con garantías de éxito. En síntesis, como meta última, se pretende promover la inserción social y laboral de este colectivo a través de la educación. Se intenta aminorar el riesgo social que pudiera producir su exclusión del mundo universitario. Los inmigrantes que cursan estudios superiores constituyen el grupo diana objeto de estudio.

En un plano más concreto, este trabajo pretende identificar las principales características que conforman el perfil del inmigrante universitario, lo que propiciará una mejor comprensión de este grupo y de sus circunstancias personales y contextuales. Esta cuestión constituye una parte esencial de la investigación aludida, al tiempo que es de gran interés pues, como es bien sabido, resulta imprescindible, antes de emprender cualquier intervención socioeducativa de calidad, conocer y analizar los mencionados rasgos.

Se ha tenido especial interés en que sean los inmigrantes los auténticos protagonistas, involucrándolos en los diferentes momentos del proceso investigador, recogiendo sus motivaciones, necesidades y problemas para cursar estudios superiores. Se pretende captar la realidad en la que viven desde todas sus ópticas y dimensiones, a fin de elaborar propuestas y medidas transformadoras que les ayuden a romper barreras, favorezcan el éxito académico y el disfrute de la equidad y justicia social.

Los resultados de los trabajos realizados anteriormente por el equipo de investigación en otros niveles educativos sobre "Diversidad cultural e inclusión social" (2003), pusieron de relieve que para lograr una integración eficaz es preciso acercarse a su cultura (historia, estilo de vida, valores, costumbres...), con el fin de comprender mejor su realidad diferencial. Así mismo, se puso de manifiesto la necesidad de diseñar medidas que favorezcan el proceso de aprendizaje. Con esta finalidad, se elaboró un modelo de intervención educativa de carácter integrador. Estas experiencias han animado a seguir esta línea en el ámbito universitario.

Ante la insuficiencia de trabajos sobre la población inmigrante en la universidad, se espera que los resultados de este estudio, constituyan un punto de partida para avanzar en hacer efectiva la igualdad de oportunidades.

\section{Metodología}

En el campo de la educación, en el que se enmarca este trabajo, la investigación va más allá de la recogida de información y su análisis descriptivo. Debe llegar a la óptica prescriptiva e identificar cuestiones realmente importantes que sean susceptibles de mejora. En otras palabras, la investigación en educación pretende conocer y estudiar la realidad para transformarla y mejorarla. Como se ha señalado, la identificación del perfil del alumnado inmigrante, ayudará a establecer los puntos diana 
que clarificarán sus necesidades más urgentes, que serán la base para diseñar buenas prácticas. Este objetivo, escasamente analizado hasta el momento, es motivo suficiente para realizar una investigación de estas características, pues, los resultados de la misma pueden contribuir a la inclusión de este colectivo en la sociedad y a su éxito académico.

Este estudio surge de la inquietud por dar respuesta a las siguientes interrogantes: ¿Qué señas de identidad se manifiestan en el colectivo? ¿Qué nivel educativo existe en el entorno familiar? ¿Qué causas les han Ilevado a inmigrar? ¿Cuáles son sus expectativas? ¿Qué les motiva a realizar estudios universitarios? ¿Tienen una buena base de conocimientos para afrontar este reto? ¿Qué les lleva a abandonar sus estudios? Tomando como base estas cuestiones, se han formulado los siguientes objetivos:

- Identificar el perfil de la población inmigrante universitaria.

- Determinar sus características personales y socioculturales.

- Examinar los motivos que les impulsan a realizar estudios universitarios en nuestro país.

- Analizar sus intereses, expectativas y antecedentes académicos.

- Diagnosticar las causas de abandono.

- Proponer líneas de acción de acuerdo con su perfil específico, con el fin de propiciar su éxito académico e integración social.

Para alcanzar los citados objetivos, se ha utilizado un enfoque multiparadigmático, con el fin de acercarnos de forma diversa y complementaria a la realidad estudiada. Así pues, se ha recurrido tanto a la metodología de carácter cuantitativo como cualitativo, para describir, comprender y explicar las variables que inciden en el perfil de los inmigrantes en la universidad.

Esta investigación cabe calificarla como no experimental, de carácter descriptivo y correlacional. Pretende analizar la situación existente en el momento en que se realiza el trabajo de campo. La unidad de análisis es el estudiante inmigrante matriculado en una carrera de una universidad pública española, bien en la modalidad presencial o a distancia.

La selección y la definición operativa de las variables constituye un momento importante en todo proceso investigador, pues deben traducirse a un lenguaje observable y cuantificable, de tal forma que la recogida posterior de información esté perfectamente delimitada. En esta investigación se pueden señalar las siguientes dimensiones o variables independientes y su incidencia en los resultados del estudio. Éstas contribuirán a definir con mayor precisión el perfil del inmigrante universitario.

- Dimensión personal y social:

- Edad y sexo.

- Expectativas personales, sociales y laborales.

- Estudios realizados previamente.

- Aspiraciones académicas. 


\section{- Dimensión contextual}

- Entorno familiar: nivel de estudios de los padres.

- Causas sociales, políticas y económicas que conducen a la emigración.

- Elección del país de acogida.

- Dificultades encontradas.

\section{- Dimensión académica}

- Motivos para matricularse en la universidad.

- Tiempo de dedicación al estudio.

- Dificultades en el estudio.

- Abandono.

- Satisfacción.

Para la recogida de información se recurrió, en primer lugar, al análisis documental para conocer en profundidad el estado de la cuestión y la situación de los inmigrantes en el mundo universitario. Así mismo, se elaboró un cuestionario de escala tipo Likert sobre las variables mencionadas, de cuatro opciones; no se eligieron cinco alternativas para evitar la tendencia a seleccionar la posición central. Dicho cuestionario constó de 41 ítems. Se utilizaron dos procedimientos diferentes para la recogida de información: aplicación directa en el aula y a través de correo electrónico.

También se confeccionó un guión previo para realizar entrevistas semiestructuradas, a fin de profundizar en los aspectos de mayor relieve que presentaran los datos cuantitativos. Dichas entrevistas han permitido penetrar en las vivencias, sentimientos y, en suma, en su vida como miembros de la comunidad universitaria. Se aplicaron a cinco alumnos de primer ciclo y a otros tres de segundo ciclo en cada una de las universidades públicas de las tres comunidades autónomas con mayor número de inmigrantes: Madrid, Cataluña y Andalucía, así como en la UNED.

En cuanto a la fiabilidad y validez del cuestionario se estimó conveniente utilizar la validez de contenido por considerarla la más adecuada. Con ello, se pretende reducir al máximo las posibles fuentes de error, recurriendo al juicio de expertos. El instrumento presentó una gran consistencia interna. La fiabilidad se calculó mediante el coeficiente de alfa de Cronbach, a fin de hallar la consistencia interna obteniéndose un valor de 0,857 que se considera adecuado. En el instrumento de carácter cualitativo también se calculó la validez y fiabilidad a través de la triangulación y del contraste intersubjetivo.

La población o universo de este trabajo está integrada por todos los inmigrantes matriculados en estudios universitarios en $1^{\circ}$ y $2^{\circ}$ ciclo que, según el Consejo de Coordinación Universitaria del Ministerio de Educación y Ciencia, tal como se ha citado anteriormente, está constituida por 29.627 sujetos, sin contar los estudiantes Erasmus.

Tal como se constata en los datos ofrecidos anteriormente, las comunidades autónomas cuyas universidades públicas presenciales cuentan con un número superior a los dos mil inmigrantes matriculados son, por orden de mayor a menor: Madrid, Cataluña, Andalucía, Comunidad Valenciana, Castilla y León. Por otro lado, dentro de los 
estudiantes que cursan sus titulaciones en universidades no presenciales, destaca la UNED, en la que se han "multiplicado por cinco el número de extranjeros en los últimos cuatro años", según el estudio realizado por el profesor Luis Camarero, información publicada el día 12 de febrero de 2008, en la prensa.

Se trabajó con una muestra de sujetos matriculados en la Universidad Autónoma de Madrid, de la Universidad Autónoma de Cataluña, la Universidad Pablo Olavide de Sevilla y la UNED. La elección de universidades con metodologías diferentes (presencial y a distancia) permitió captar la problemática con mayor amplitud, al tiempo que diseñar acciones de mejora en ambas metodologías.

Para seleccionar la muestra representativa de cada centro universitario se aplicó la fórmula para poblaciones finitas de Bugeda (1974). Se realizó un muestreo estratificado cuyas unidades de análisis se seleccionaron de forma aleatoria. Teniendo en cuenta estos criterios, Tagliacarne (1968) fija la cantidad de la muestra en 400 sujetos. Para mitigar los previsibles errores y deserciones que se suelen producir, se amplió la muestra hasta 1.890 alumnos, recibiendo una respuesta adecuada de 453 personas, cifra que en todo caso supera la establecida. Este valor representa un $24,62 \%$ de estudiantes inmigrantes que respondieron a la encuesta, dato que puede considerarse satisfactorio en los envíos masivos. Del mismo modo, estos datos pueden considerarse representativos, aunque con limitaciones, del colectivo de estudiantes inmigrantes en la universidad española.

Para el análisis y tratamiento de los datos de carácter cuantitativo se utilizó el paquete informático SPSS (Statistickal Package for Social Sciences), en su versión 17.0, por considerarlo especialmente adecuado. Para el tratamiento de los datos cualitativos recogidos mediante las entrevistas semiestructuradas se utilizó el programa ATLAS.TI 5.2. Se ha seleccionado este programa por su compatibilidad, amigabilidad y funcionalidad con el paquete informático SPSS. El proceso de análisis lo desarrolla en dos fases (textual y conceptual) estrechamente interconectadas. Permiten establecer relaciones entre elementos, construir redes y representaciones gráficas facilitando la interpretación.

Se realizaron análisis de tipo descriptivo que han permitido conocer las características identificativas del conjunto de sujetos y análisis de tipo inferencial, que proporcionan una visión más global y completa a fin de derivar conclusiones probabilísticas y establecer diferencias significativas.

\section{ANÁLISIS Y DISCUSIÓN DE LOS RESULTADOS}

El análisis y la discusión son dos elementos importantes a tener en cuenta en cualquier proceso de investigación. Ambos se desarrollan en el presente apartado, a fin de ofrecer una visión panorámica de los resultados más sobresalientes, gracias a los análisis efectuados tanto de índole cuantitativa como cualitativa. El estudio de los mismos ha tenido por finalidad lograr una interpretación lo más completa posible. Ello ha permitido construir un perfil general del inmigrante universitario, aportación novedosa y de gran interés.

Si nos preguntamos cuáles son los rasgos singulares del inmigrante, los datos obtenidos revelan que coinciden, en gran medida, con el autóctono. En su mayoría 
es joven, pues se encuentra en una franja de edad entre los veinte y los treinta años $(41,18 \%)$, mientras que los que superan los cuarenta constituye un porcentaje bastante menor $(26,70 \%)$, tal como se muestra en el gráfico siguiente:

Gráfico 1. Edad.
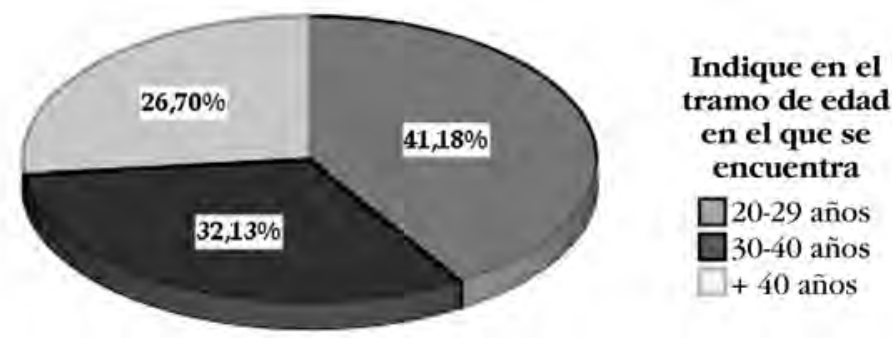

Otro rasgo que los caracteriza es el fuerte predominio del sexo femenino situado en el $71,27 \%$. Este último dato pone de manifiesto que, si bien la mujer se ha incorporado a la universidad más tarde que el varón, en estos momentos se ha roto dicha tendencia superando todas las previsiones. Ello supone un importante punto de inflexión con amplias repercusiones en todos los campos de la vida personal, social, económica y cultural. Revela que la mujer se haya especialmente motivada para superar su situación buscando, a través de los estudios, una vía de promoción social.

Los resultados de la investigación aportan información suficiente para elaborar un perfil diferencial significativo entre mujer y hombre. Las primeras consideran los estudios como fuente de promoción personal y profesional. Aprecian, singularmente, la perspectiva de utilidad de los recursos educativos. Financian sus estudios, preferentemente, gracias a la familia y a las becas, que obtiene en un número superior. Valoran, especialmente, la orientación de los compañeros de cursos superiores y la utilización de protocolos de exámenes. Los segundos, suelen dedicar más de quince horas semanales al estudio. Emigran de su país, principalmente, por la ausencia de servicios esenciales y coinciden con las mujeres al señalar causas como: obtener un porvenir mejor a través de los estudios y conseguir un trabajo adecuado a su formación. Por otro lado, se distinguen por sufragar sus estudios a través del trabajo y por mostrar menores expectativas de finalizar la carrera y de ampliar su formación.

Las variables sexo y edad muestran un comportamiento similar al presentado en el último informe 2008-2009 elaborado por el Ministerio de Ciencia e Innovación relativo a la población universitaria: la presencia mayoritaria de la mujer en las aulas, así como del alumnado joven, menor de treinta años.

Todos sostienen que sus expectativas personales, sociales y laborales se centran en la búsqueda de una mejor calidad de vida. La información de carácter cualitativo permite afirmar que anhelan ampliar, mejorar y fortalecer su nivel cultural y obtener una titulación superior en un país ajeno al suyo, para mejorar su imagen al retornar al 
mismo. Destacan, especialmente, sus expectativas laborales, pues lo que más les preocupa es encontrar un trabajo digno o bien promocionar en su ejercicio profesional.

En esta misma línea, otros estudios vienen corroborando la lógica subyacente en los planteamientos expuestos. En ellos, se evidencia que, a mayor nivel de formación, existen más posibilidades de progreso sociolaboral, en especial en los tiempos de crisis en los que el acceso al empleo resulta especialmente competitivo. Estos datos refuerzan la importancia que está adquiriendo el aprendizaje a lo largo de la vida y la formación continua puesto que muchos inmigrantes universitarios concilian el estudio y el trabajo. Esta información contrasta con el perfil general de los estudiantes españoles reflejado por el citado Informe del Ministerio, en el que se pone de manifiesto que el $67,4 \%$ no compaginan trabajo y estudio. Esta es, por lo tanto, una variable diferencial de gran interés.

Una amplia mayoría, el 63,2\% accedió a la universidad a través de estudios previos como el Bachillerato Unificado y Polivalente (BUP), el Bachillerato LOGSE o la Formación Profesional de Tercer Ciclo. Con índices mucho menores (sobre el 18\%) ingresó gracias a la convalidación de estudios realizados en su país, y, en último lugar, el 17,2, por medio de la prueba de acceso para mayores de 25 años. Predominan, por lo tanto, los cauces habituales presentes en la población autóctona.

El hecho de vivir fuera de su país parece no condicionar sus aspiraciones académicas puesto que se matriculan, preferentemente, en licenciaturas $(53,1 \%)$ e, incluso, aunque en escaso número, en postgrados. Conviene destacar que la mayoría de los encuestados se sienten capacitados para terminar sus estudios universitarios.

Tabla 2. Estudios realizados en la Universidad.

\begin{tabular}{|l|l|c|c|c|c|}
\hline Estudios & Frecuencia & Porcentaje & $\begin{array}{c}\text { Porcentaje } \\
\text { válido }\end{array}$ & $\begin{array}{c}\text { Porcentaje } \\
\text { acumulado }\end{array}$ \\
\hline \multirow{3}{*}{ Válidos } & Diplomatura & 170 & 37,9 & 37,9 & 37,9 \\
\cline { 2 - 6 } & Licenciatura & 238 & 53,1 & 53,1 & 100,0 \\
\cline { 2 - 6 } & $\begin{array}{l}\text { Doctorado y } \\
\text { Máster }\end{array}$ & 40 & 8,9 & 8,9 & 46,9 \\
\cline { 2 - 6 } & Total & 448 & 100,0 & 100,0 & \\
\hline
\end{tabular}

Llama la atención el salto generacional tan acusado que se ha producido en un lapso de tiempo tan breve. Prácticamente, la mitad de los encuestados (44,2\%) ya había realizado otros estudios universitarios en su país de origen. Esta situación contrasta claramente con la de sus padres, puesto que tan sólo, un bajo porcentaje de ellos, logró dicho nivel (17,2\% de las madres y el 23,4\% de los padres). Por otro lado, persiste un índice importante de progenitores sin estudios (34,8\% de las madres y el $33,3 \%$ de los padres), valores muy elevados frente a los parámetros que presenta la población española, donde los que no tienen estudios apenas alcanzan el $10 \%$. Se puede constatar, una vez más, que el nivel educativo alcanzado por las madres es lige- 
ramente más bajo. Tal como se ha apuntado, se está logrando un ascenso considerable en el nivel de estudios de los hijos respecto a la generación anterior. Puede ser debido a una mayor toma de conciencia sobre la importancia de la educación.

La causa principal que les ha llevado a emigrar de su país ha sido buscar un porvenir mejor, a través de la realización de estudios universitarios y, también, aunque en menor medida, por la escasa remuneración laboral y la falta de acceso y de cobertura de servicios esenciales. Nos encontramos pues ante un grupo de personas que dejan su país, fundamentalmente, por problemas de índole económica. Este tipo de inmigración es la más frecuente en la actualidad. Como sostiene en su investigación Ruiz Olabuénaga (2000) los inmigrantes típicos, los más numerosos, son aquellos sujetos cuyo motivo básico es la búsqueda de trabajo o de asilo político.

No es de extrañar que el idioma compartido aparezca como un factor decisivo a la hora de elegir nuestro país en concreto. Constituye un aspecto de singular importancia que contribuye de forma decisiva a la integración social y laboral. La lengua es un vehículo de comunicación esencial que facilita las relaciones humanas y reduce la incertidumbre ante situaciones desconocidas. Cuando Ilegaron se encontraron con dificultades, en muchos casos no previstas, entre las que se pueden mencionar, por orden de importancia: el acceso a un empleo, a la vivienda y los problemas de carácter legal.

Las razones que les han Ilevado a matricularse en la universidad española, ponen de relieve, sobre todo, los aspectos culturales y de formación. En este sentido, les preocupa por orden de importancia: la obtención de una titulación europea, seguido de cerca por la ampliación de los estudios realizados anteriormente y la de conseguir mejoras laborales y, en menor medida, legalizar un título.

Tabla 3. Causas de matricularse en la Universidad española.

\begin{tabular}{|l|c|c|}
\hline & Valores medios & Desviación típica \\
\hline Obtención de la titulación universitaria & 3,32 & 1,056 \\
\hline Ampliación de los estudios anteriores & 3,31 & 1,050 \\
\hline Mejoras laborales & 2,87 & 1,183 \\
\hline
\end{tabular}

La información aportada por el análisis cualitativo permite añadir otros aspectos sobre esta cuestión como: conseguir un mayor prestigio personal y social, un status sociolaboral más elevado y el hecho de que las titulaciones españolas están bien valoradas.

La dedicación al estudio fuera de la universidad no es muy elevada, posiblemente por el amplio horario laboral al que están sujetos. Algo más de un tercio $(37,9 \%)$ dedican menos de 10 horas semanales aunque, un porcentaje apreciable $(21,4 \%)$, invierte en esta actividad más de 15 . En cuanto al compromiso académico se comportan responsablemente. Como se desprende de los datos obtenidos, los 
alumnos o bien no se presentan a examen (22,3\%) o los que se presentan, lo hacen bien preparados, superando las asignaturas.

El proceso formativo iniciado no se haya carente de dificultades educativas exógenas y endógenas. Ambas se interrelacionan siendo prácticamente imposible identificar la línea de separación entre ellas. En coherencia con lo expuesto, señalan como lo más complejo el conciliar los estudios con el trabajo; seguido de los problemas administrativos y de gestión. Entender las explicaciones del profesor les supone una dificultad menor, si bien valoran muy positivamente el apoyo recibido del mismo. Entre otros problemas a afrontar se encuentran: adquirir hábitos de trabajo intelectual, aprender a organizar su tiempo, saber buscar la información que necesitan, estructurar debidamente el trabajo autónomo, realizar los exámenes, así como aprender a concentrarse para obtener el máximo rendimiento en el estudio. Ponen de relieve, del mismo modo, el dominio de la tecnología y el no poder asistir a clase de forma regular. La información de carácter cualitativo, relaciona los códigos primarios elaborados a partir de los vocablos e ideas expresadas por las respuestas de los estudiantes. En la siguiente red, se muestran la categoría principal y el resto de los códigos resultantes de la primera codificación.

Gráfico 2. Dificultades educativas.

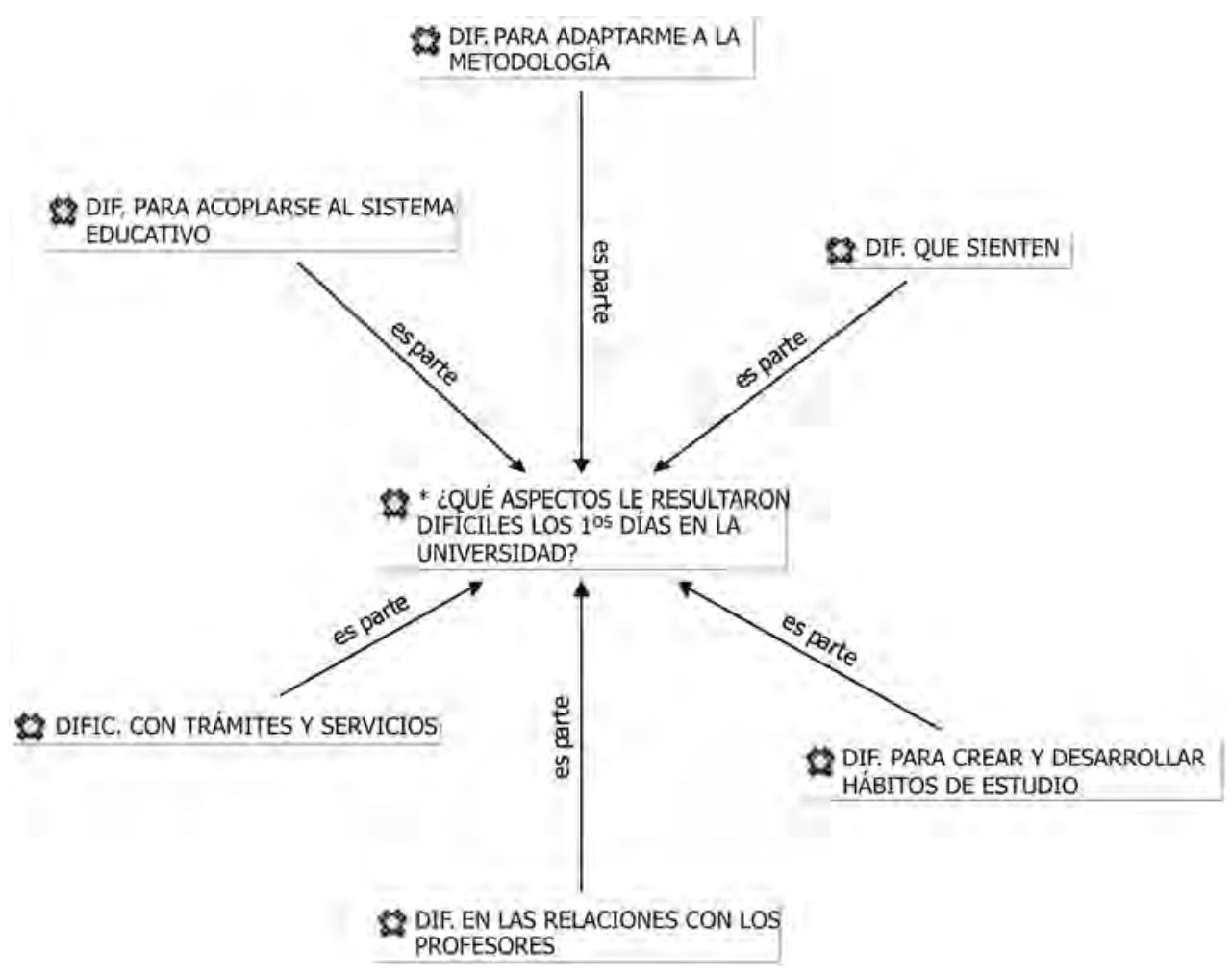


Cabe señalar que, en general encuentran problemas para adaptarse al sistema educativo español, al desconocer el funcionamiento interno de la universidad, en los aspectos académicos, metodológicos, de evaluación y de profesorado. No es de extrañar, que en los primeros días, se sientan perdidos y desorientados en un lugar totalmente nuevo, lo que contribuye a incrementar la ansiedad ante lo desconocido.

Otra cuestión relevante se refiere al abandono de los estudios que se hace presente, en la mayor parte de las ocasiones, por motivos personales, laborales y económicos. Al profundizar en esta temática nos encontramos con otros aspectos metodológicos-didácticos que mencionan los propios protagonistas, como la percepción de que: los profesores no les motivan suficientemente; no se esfuerzan por hacerles asequible el programa y no realizan simulacros de exámenes y de tareas. También, señalan que las tutorías son muy escasas, así como la ausencia de tutorías virtuales para poderlas seguir en horario disponible. Del mismo modo, aluden a causas como las cargas familiares, la exigencia de los trabajos, la amplitud del temario y la falta de competencias previas.

En el abandono inciden también los sentimientos personales como el experimentar soledad, cansancio, frustración y, en muchas ocasiones, agobio al tener que superar un determinado número de créditos para la renovación del permiso de estancia en el país. No suelen acudir a los Centros de Orientación e Información y Empleo (COIEs) puesto que desconocen sus funciones e incluso su existencia. Sin embargo, estas entidades podrían desempeñar una importante labor en orden a reducir el abandono. En general, estamos ante un grupo satisfecho con nuestra institución universitaria, pues la consideran promotora de políticas de inclusión social y prácticamente la totalidad estaría dispuesto a matricularse de nuevo.

Dibujado el perfil del inmigrante universitario, se está en condiciones de abordar una serie de conclusiones y propuestas con el fin de mejorar la inclusión social de este colectivo.

\section{CONCLUSIONES Y PROPUESTAS}

Este trabajo ha pretendido en todo momento, hacer visible los intereses, necesidades y demandas de este colectivo. Trata de recoger la voz de los protagonistas, con el fin de profundizar en los diferentes aspectos que afectan a su vida universitaria y conforman su perfil diferencial, perfil que no es fácil de definir, dado que no estamos ante una población homogénea. En las siguientes líneas se destacan los rasgos más significativos.

En primer lugar, se debe resaltar que la presencia del alumnado inmigrante, especialmente el no comunitario, escasamente representado en décadas anteriores, se está haciendo cada vez más visible, alcanzando cifras significativas en la universidad. Una de las posibles causas es que, al número habitual de estudiantes extranjeros hay que añadir el colectivo denominado de "segunda generación". Esta afirmación se sustenta en los datos aportados por este trabajo que ponen en evidencia que más de la mitad accede a la universidad habiendo realizado el itinerario común en el sistema educativo español. 
El inmigrante universitario-tipo, se caracteriza, por ser joven y del sexo femenino. Compatibiliza estudio y trabajo, situación menos frecuente en el alumnado autóctono. Así mismo se siente capacitado para finalizar su carrera, a la vez que aceptado por sus compañeros.

La búsqueda de un porvenir mejor, cuya vía de acceso más importante es la educación superior, la problemática laboral existente y la ausencia de servicios básicos, son las causas principales de la emigración. El idioma compartido ha contribuido de forma decisiva a elegir nuestro país. A su llegada han de resolver dificultades relacionadas con su integración en el mercado laboral, el acceso a una vivienda digna, así como regularizar su situación legal.

Las exigencias actuales del mercado laboral, demandan mano de obra cualificada, lo que impulsa a esta población a efectuar estudios de larga duración. Conviene reseñar que se ha producido un importante progreso sociocultural, al elevarse considerablemente el nivel de estudios de los hijos respecto a sus padres. Una parte importante de los primeros Ilegan a España habiendo realizado ya una carrera universitaria, por lo que nos encontramos con un grupo cualificado. Eligen la universidad para continuar su formación, mejorar su empleo y conseguir legalizar su título. Financian sus estudios a través de su trabajo y, en menor medida, gracias a la familia y las becas.

Intereses y expectativas mantienen una clara asociación. Por ello, se hace alusión, a las segundas que recogen, en cierta medida, las inquietudes y las aspiraciones del alumnado. Dichas expectativas, en líneas generales, son elevadas centrándose en finalizar la carrera y conseguir un trabajo acorde con la formación adquirida. Del mismo modo, desean ampliar, mejorar y fortalecer su nivel cultural y obtener una titulación superior en un país europeo de modo, que al regresar al suyo, sean mejor considerados y reconocidos. Destacan, igualmente, las relativas a la mejora y promoción laboral. En cuanto a las de carácter personal y social las centran, fundamentalmente, en el logro de una mayor calidad de vida.

Todas las expectativas se hallan muy interrelacionadas con el resto de variables estudiadas, en especial con los intereses en la elección de carrera y con la integración en el país. Sin embargo, se hallan menos vinculadas con las causas de abandono y con las dificultades encontradas los primeros días en el centro educativo. En general, la mujer se inclina más por las expectativas de tipo académico y laboral, mientras que la posibilidad de retornar a su país es más apreciada por el hombre.

El abandono es un problema importante en este colectivo debido a la situación vulnerable en la que se encuentra. La causa principal por la que suelen dejar de estudiar radica en la dificultad que supone conciliar el estudio y el trabajo, seguida de las asociadas a la organización y desarrollo del currículo, la mala autogestión del tiempo, así como a cuestiones personales vinculadas con el mundo afectivo. Otras se relacionan con una preparación académica insuficiente para seguir con regularidad las clases, con la carencia de técnicas de trabajo intelectual y con cuestiones sociales, económicas y laborales.

La integración es un factor esencial en el éxito académico. La vida universitaria genera ambientes y espacios de encuentro, en los que los sujetos pueden interaccionar y potenciar la cercanía entre personas de diferentes culturas y formas de pensar. Un indicio de singular importancia que permite ratificar la citada integración, se 
apoya en el hecho de que se muestran satisfechos tanto en nuestro país, como en la institución universitaria, dado que no contemplan en sus planes de vida inmediatos el regreso al suyo y están claramente decididos a continuar estudios en el nuestro.

La labor de los Centros de Orientación Información y Empleo (COIEs) existentes en las universidades, como se ha indicado, es poco visible para este colectivo, dado que los desconocen y, por lo tanto, son escasamente valorados. Queda pues una labor importante a desarrollar por parte de estos centros a fin de prestar los servicios para los que han sido creados. Un reto importante lo constituye la atención a la población inmigrante, en general, especialmente necesitada de asesoramiento.

A modo de síntesis, la información obtenida en esta investigación pone de relieve que estamos ante un colectivo cada vez más numeroso que presenta un perfil singular con intereses y necesidades específicas, que reclaman medidas de apoyo diferenciadas y personalizadas que propicien el éxito académico y su integración en la sociedad.

Tomando como referencia la sugerencia de Bueno Aguilar (1999: 58) sobre la necesidad de que en cualquier propuesta formativa se tenga en cuenta que en la sociedad actual ha de primar el intercambio y la comunicación entre distintas culturas, se han elaborado las propuestas que se exponen a continuación. Las mismas tienen por objeto proporcionar a los inmigrantes universitarios los recursos necesarios para lograr su autonomía en el ejercicio de derechos y deberes, en condiciones similares a las de la población autóctona, así como su mejor inclusión en la sociedad:

- Transformar la universidad en espacio de comunicación, convivencia e integración en el entorno, promoviendo actividades de extensión universitaria de carácter intercultural que fomenten el conocimiento y el respeto de los valores y normas sociales de tolerancia y convivencia. Es importante crear ambientes educativos, superadores de estereotipos y prejuicios que propicien la valoración de la diferencia y el diálogo.

- Sensibilizar a la comunidad universitaria con el fin de que se fomente, a través de los planes de estudio, la adquisición de competencias interculturales. Igualmente, se suscitará el rechazo de cualquier manifestación de discriminación, racismo y xenofobia en todos los campos de la vida social, tanto en el ámbito público como privado.

- Establecer una red interuniversitaria a través de las tecnologías de la información y la comunicación, que propicie, en todo el territorio, la interrelación del alumnado inmigrante proveniente de diferentes culturas. Esta red deberá estar abierta a todos los alumnos, si bien se potenciará la creación de canales específicos por carreras, con el fin de favorecer la comunicación, el intercambio de conocimientos y el apoyo académico y socio-afectivo.

- Ofrecer herramientas didácticas y recursos personalizados tales como: el conocimiento de diferentes metodologías y modelos de aprendizaje; cursos de técnicas de trabajo intelectual y material didáctico en soportes variados (libros en formato texto y electrónico, manuales, guías, videos y audios). Mención especial merece la Guía de competencias para el estudio que dotará al estudiante de habilidades y destrezas para la comprensión del conocimiento y la Guía Didáctica Interactiva en Tecnologías de la Información y Comunicación que facilitará la utilización de las mismas para mejorar el aprendizaje. 
- Desarrollar, a través de los Centros de Orientación, Información y Empleo (COIEs) de las universidades, las siguientes acciones:

- Elaborar un diagnóstico personalizado sobre los factores a los que se debe prestar atención para que los inmigrantes finalicen con éxito sus estudios.

- Prevenir el fracaso diseñando diferentes líneas de acción desde los primeros cursos. Entre ellas, se puede mencionar el sistema de acogida de los alumnos nuevos y de todos aquellos que se encuentren en situación de vulnerabilidad. Dicho sistema colaborará con los estudiantes a través de actividades diversas: organización de jornadas de bienvenida en las que se presentarán los diferentes servicios de la universidad y se darán a conocer los derechos y deberes del estudiante universitario, así como las exigencias de aprendizaje y rigor científico del plan de estudios.

- Potenciar el acompañamiento a través de la ayuda entre pares para implicar a los alumnos de cursos superiores, inmigrantes y autóctonos. Por medio de este procedimiento, todos podrán desempeñar una labor de asesoramiento, información y apoyo de los alumnos nuevos (pedagógicamente se conoce como alumno-mentor). Los estudiantes acompañantes o mentores recibirán créditos de libre configuración por realizar esta función.

- Fortalecer la figura del Defensor del Estudiante al que pueden acudir todos los que lo precisen y, especialmente, los de origen extranjero. Este servicio tiene como misión velar y garantizar los derechos y deberes de este colectivo. Entre sus funciones se encuentran el asesoramiento y apoyo en los diferentes aspectos en los que lo precisen; en especial para efectuar los trámites burocráticos, informar sobre las ayudas al estudio y de los requisitos para legalizar y homologar titulaciones académicas.

Este conjunto de medidas facilitarán la inserción del inmigrante en la universidad a la vez que evitará el sentimiento de soledad, inquietud y ansiedad ante situaciones nuevas.

El desafío que supone el aumento progresivo del flujo de inmigrantes en la universidad, resulta difícil de abordar con éxito en un momento de crisis, tanto por la falta de recursos como por la necesidad de una formación específica del profesorado, como elemento clave en la educación intercultural. Existe una escasa percepción de las implicaciones educativas que conlleva la realidad multicultural en las aulas, por lo que los docentes apenas consideran necesario introducir innovaciones curriculares y metodológicas. Es preciso impulsar una pedagogía de la inclusión que se empeñe en formar en una dinámica de solidaridad que reconozca la causa de los otros, que acepte trabajar desde la valoración y el respeto, que crea en la dignidad de las personas y luche por eliminar falsas representaciones, prejuicios y discriminaciones.

Para concluir, se subraya la necesidad de favorecer la comprensión por parte de la sociedad del fenómeno migratorio con el fin de mejorar la convivencia intercultural, valorando la pluralidad y fomentando la tolerancia. Al mismo tiempo, es preciso caminar hacia una universidad inclusiva en la que convivan las diferentes culturas y contribuya a la formación integral de las personas, a fin de que sean capaces de construir su identidad en la diversidad. 


\section{REFERENCIAS BIBLIOGRÁFICAS}

AGUADO ONDINA, Mำ T. (2006). Educación intercultural. Necesidades de formación del profesorado desde una perspectiva europea. Madrid: UNED Ediciones. ÁLVAREZ RODRÍGUEZ, A. (2006). Inserción e integración laboral de los inmigrantes: políticas e instrumentos. Valencia: Alfa Delta Digital S. L.

ARGULLOL, E. y LÓPEZ, G. (2004). Inmigración y transformación social en España: Madrid: Generalitat de Catalunya, Fundación BBVA y el Instituto de Estudios Autonómicos.

BANKS, J. A. (1994). An introduction to multicultural education. Boston: Allyn and Bacon.

BUGEDA, J. (1974). Manual de Técnicas de Investigación Social. Madrid: Instituto de Estudios Políticos.

BORGSTRÖM, M. (2002). Hacia una Europa diferente. Respuestas educativas a la interculturalidad. Madrid: CIDE/CIDREE/SLO.

BUENO AGUILAR, J. J. (1999). Somos una sociedad multicultural, canon educativo. En Pedagogía Social. Revista Interuniversitaria, 3, segunda época, 57-71.

CAVALCANTI, L. (2007). Los vínculos económicos y familiares transnacionales: los inmigrantes ecuatorianos y peruanos de España. Madrid: Fundación BBVA.

CONSEJO DE COORDINACIÓN UNIVERSITARIA (2006). Datos y Cifras del Sistema Universitario Español. Curso 2005-06. Disponible en: http://www.educacion.es/ $\mathrm{dctm} / \mathrm{mepsyd} /$ educacion/universidades/estadisticas-informes/datos-generales/datos05-06.pdf?documentld=0901e72b80048a9b (consultado 19/09/2009).

CONSEJO DE COORDINACIÓN UNIVERSITARIA (2007). Datos y Cifras del Sistema Universitario Español. Curso. 2006-07. Madrid: MEC.

CONSEJO DE COORDINACIÓN UNIVERSITARIA (2008): Datos Básicos del Sistema Universitario Español. Curso 2008-09. Disponible en: http://www.oei.es/ salactsi/Informe2008-2009.pdf (consultado 22/09/2009).

DELORS, J. (1996). Informe a la UNESCO, Aprender para el siglo XXI. La educación encierra un tesoro. Madrid: Santillana-UNESCO.

DÍEZ NICOLÁS, J. (2007). La segunda generación de inmigrantes: una aproximación al caso español. Madrid: Ayuntamiento de Madrid. Observatorio de Seguridad.

EUROSTAT-COMISIÓN EUROPEA (2003). La situación social en la Unión Europea 2002. Disponible en: http://www.europa.eu.int/comm/eurostat.

FORO DE ALMAGRO (2006). Criterios y directrices para la garantía de la calidad de las universidades en el EEES: VII Foro de Almagro, 24 y 25 de octubre de 2005. Ciudad Real: Ediciones de la Universidad de Castilla-La Mancha.

GARCÍA LLAMAS, J. L., PÉREZ SERRANO, G., SARRATE CAPDEVILA, Mํa L. y otros (2003). Diversidad cultural e inclusión social. Salamanca: Témpora.

INSTITUTO NACIONAL DE ESTADÍSTICA (2008): Revisión del Padrón Municipal a 1 de enero de 2008. Disponible en: http://www.skyscrapercity.com/showthread.php?t= 774554 (consultado 2/10/2009).

MANZANO ARRONDO, V. y ANDRÉS ZAMBRANA, L. (2007). El diseño de la nueva universidad europea. Sevilla: Atrapasueños. 
MANZANO CORTÉS, M. E (2007). Integración de alumnos y alumnas inmigrantes. Almería: Asociación Procompal de Profesores.

MINISTERIO DE TRABAJO Y ASUNTOS SOCIALES (2007). Plan (2007-2010) estratégico de Ciudadanía e Integración. Madrid: Subdirección General de Información Administrativa y Publicaciones.

MORIÑA, A. y RIÑA, A. (2004). Teoría y Práctica de la educación inclusiva. Málaga: Aljibe.

NACIONES UNIDAS (2007). Previsiones demográficas mundiales. Revisión de 2006. Resumen. Nueva York: Naciones Unidas, Departamento de Asuntos Económicos y Sociales. División de Población.

NACIONES UNIDAS (2009): Informe sobre Desarrollo Humano 2009 "Superando barreras: Movilidad y desarrollo humanos". Nueva York: Programa de Naciones Unidas para el Desarrollo.

PÉREZ SERRANO, G., SARRATE CAPDEVILA, Ma L., QUICIOS, P. GARCIA y otros (2009). Hacia una universidad inclusiva. Medidas de apoyo al inmigrante universitario. Madrid: Ministerio de Ciencia e Innovación. Disponible en: www.ea-web.es (consultado 5/10/2009).

QUICIOS GARCÍA, Ma del P. y FLORES RAMOS, E. (2005). Población inmigrante: su integración en la sociedad (una visión desde la educación). Madrid: Pearson.

RUIZ OLABUÉNAGA, J. I. (2000). Inmigrantes. Madrid: Acento.

SIMÓN, G. (2002). "Les migrations internationals", Population et Societés, 382, septiembre.

SORIANO AYALA, E. (Coord.) (2005). La interculturalidad como factor de calidad educativa. Madrid: Muralla.

TAGLIACARNE, G. (1968). Métodos para la investigación de mercado. La muestra. Barcelona: Ariel.

VARIOS AUTORES (2002). Educación inclusiva. Monográfico. Revista de Educación, 327. 